\title{
Correction to: Temperature tunable like-electromagnetically induced transparency in metasurface with vanadium dioxide
}

\author{
Renxia Ning $^{2,3}{ }_{(D)}$, Zhinqiang Xiao ${ }^{1}$, Zhenhai Chen ${ }^{2,3}$, Wei Huang ${ }^{1, a}$ \\ ${ }^{1}$ State Key Laboratory of ASIC and System, Shanghai Institute of Intelligent Electronics and Systems, School \\ of Microelectronics, Fudan University, Shanghai 200433, China \\ 2 School of Information Engineering, Huangshan University, Huangshan 245041, China \\ ${ }^{3}$ Engineering Technology Research Center of Intelligent Microsystems, Huangshan 245041, AnHui Province, \\ China
}

(C) The Author(s), under exclusive licence to Società Italiana di Fisica and Springer-Verlag GmbH Germany, part of Springer Nature 2021

\section{Correction to: Eur. Phys. J. Plus (2021) 136:751 \\ https://doi.org/10.1140/epjp/s13360-021-01164-x}

The original version of this paper was inadvertently published with an incorrect affiliation for the author Zhinqiang Xiao.

The correct affiliation is:

State Key Laboratory of ASIC and System, Shanghai Institute of Intelligent Electronics and Systems, School of Microelectronics, Fudan University, Shanghai 200433, China

The original article has been corrected. We apologise for any inconvenience caused to our readers.

The original article can be found online at https://doi.org/10.1140/epjp/s13360-021-01164-X.

\footnotetext{
a e-mail: eehuangw@fudan.edu.cn (corresponding author)
} 